\title{
Relative Contribution of Amyloid- $\beta$ Plaque Associated and Plaque Distant Microglia to Alzheimer'S Disease (AD) Progression
}

\section{Anne-Laure Hemonnot-Girard}

IGF, CNRS, INSERM, Univ. Montpellier

\section{Cédric Meersseman}

IGF, CNRS, INSERM, Univ. Montpellier

\section{Manuela Pastore}

Université de Montpellier, CNRS, INSERM, BioCampus UAR3426

Nathalie Linck

IGF, CNRS, INSERM, Univ. Montpellier

\section{Catherine Rey}

SFR santé Lyon-Est, CNRS UMR-S3453, Inserm US7

Joël Lachuer

Université Lyon 1

\section{Christelle Reynes}

Université de Montpellier, CNRS, INSERM, BioCampus UAR3426

\section{François Rassendren}

IGF, CNRS, INSERM, Univ. Montpellier

Hirbec Hélène ( $\nabla$ helene.hirbec@igf.cnrs.fr )

IGF, CNRS, INSERM, Univ. Montpellier

\section{Research Article}

Keywords: Microglia, Alzheimer disease, Amyloid-plaques, Inflammation, Laser microdissection, RNA-seq

Posted Date: December 23rd, 2021

DOI: https://doi.org/10.21203/rs.3.rs-876995/v2

License: (c) (i) This work is licensed under a Creative Commons Attribution 4.0 International License.

Read Full License 


\section{Abstract}

Background: Research in recent years firmly established that microglial cells play an important role in the pathogenesis of Alzheimer's disease (AD). In parallel, a series of studies showed that, under both homeostatic and pathological conditions, microglia are a heterogeneous cell population. In AD, amyloid-b (Ab) plaque-associated microglia (PAM) display a clearly distinct phenotype compared to plaque-distant microglia (PCM), suggesting that these two microglia subtypes likely differently contribute to disease progression. So far, molecular characterization of PAM was performed indirectly using scRNA-seq approaches or based on markers that are supposedly up-regulated in this microglia subpopulation.

Methods: In this study, we combined cell-specific laser capture and RNA-seq analysis to investigate, without preconceived notions of the molecular and/or functional changes that would affect these cells, the functional role of both plaque-associated and plaque-distant microglia.

Results: First, we established that this approach allows selective isolation of microglia, while preserving spatial information and preventing transcriptome changes induced by classical purification approaches. Then, we identified, in PAM and PCM subpopulations, networks of co-deregulated genes and analyzed their potential functional roles in AD. Finally, we investigated the dynamics of microglia transcriptomic remodeling at early, intermediate and late stages of the disease.

Conclusions: Our comprehensive study demonstrates that the proximity of microglia to Ab-plaques dramatically alters the microglial transcriptome and reveals that these changes can have both positive and negative impacts on the surrounding cells. These opposing effects may be driven by local microglia heterogeneity also demonstrated by this study. Our results also suggest that Ab plaque-associated microglia undergo exhaustion in the later stage of the disease. Our approach leads to molecularly define the overlooked plaque-distant microglia. We show that plaque-distant microglia are not bystanders of the disease, although the transcriptomic changes are far less striking compared to what is observed in plaque-associated microglia. In particular, our results suggest they are involved in Ab oligomer detection and in Ab-plaque initiation, with increased contribution as the disease progresses.

\section{Background}

Alzheimer's Disease (AD) is a progressive neurodegenerative disorder and the most common cause of dementia. It affects around 50 million people worldwide and incidence is expected to double within the next 20 years. From a histological point of view, the disease is characterized by several pathological hallmarks, including amyloid-b (Ab) plaques, neurofibrillary Tau tangles, loss of synapses, dystrophic neurites and neuro-inflammation (1). The chain of events leading to $A D$ has been the focus of intense research for decades, but remains poorly understood. Based on the sequential appearance of the hallmarks it has been proposed that Ab deposition represents an initiating event in the cascade that ultimately leads to neuronal degeneration (2). However, recent results suggested that Ab accumulation alone is insufficient to explain the disease progression (3). 
Recent human genome wide association studies (GWAS) revealed that many risk factors for AD are associated with genes that are either highly, preferentially or exclusively expressed by microglia (4-7). These data, together with the identification of profound remodeling of the microglia transcriptome in AD mouse models (5-7), support the hypothesis that microglia are key contributors to AD pathogenesis. They also open the therapeutic potential of modulating microglial functions for AD treatment. However, the role of microglia in $A D$ is still unclear and heavily debated, with conflicting reports regarding their protective or detrimental impact.

Microglia are the innate immune cells of the brain where they have key homeostatic functions. In the adult brain, they notably provide trophic support for neurons, regulate neuronal excitability, and actively monitor the brain parenchyma (8). Yet, when brain homeostasis is disrupted microglia respond. Once believed to be an all-or-nothing process, it is now well established that microglia reaction is a complex and dynamic biological process which depends on many factors including, the brain region, the sex, the nature of the trigger and the duration of the triggering event. Further, high-content molecular studies have established that both homeostatic and reactive microglia are indeed very diverse $(9,10)$. scRNA-seq studies have been instrumental in deciphering the molecular diversity of microglia in neurodegenerative disease. In AD, these approaches have allowed the identification of several microglia subtypes linked to the disease (11-13). The disease-associated microglia (DAM, (11)), the late response microglia (LRM, (12)) and the activated response microglia (ARM, (13)) appear closely related. Others microglia subtypes have also been described in AD including, the early response microglia (ERM, (12)), the interferon-response microglia (IRM, (13)), and the transiting response microglia (TRM, (13)), but their roles remain elusive.

In addition to their molecular diversity, microglia cells in AD display a clear phenotypic heterogeneity. Abplaque associated microglia, here referred to as PAM, exhibit strong morphological alterations. More specifically, they present a reduced average surface area and branching that is a morphological characteristic typical of reactive microglia (14). PAM also undergo important channel activity changes, and display altered phagocytic properties $(14,15)$. In contrast, plaque-distant microglia, here referred to as PCM, display only minor morphological alteration compared to control microglia (14). Still, in the late stage of the disease, PCM display an intermediate inflammatory profile compared to either non-AD control, here referred to as control microglia (CM), or plaque-associated microglia (16). However, in general, plaque-distant microglia have been largely overlooked in $A D$, and whether the observed subtle morphological changes are associated with functional changes in this subtype has not been clearly defined. Adding to the complexity, significant temporal diversity in microglial morphology has been observed, in both mouse models and patients, during AD progression (17).

The molecular signature of PAM has only been indirectly inferred. First, based on the expression of a handful of markers, molecularly defined DAM, ARM and LRM have been proposed to correspond to the spatially defined PAM (11-13). Second, based on initial assumptions that PAM are Class II major histocompatibility complex positive (MHCI+) (16) or are actively Ab-phagocytosing cells (18), more recent studies used Fluorescent-activated Cell sorting (FACS) to characterize the transcriptome of PAM 
compared to PCM (i.e. MHCll- or Ab-non-phagocytosing microglia). However, these latter approaches required tissue dissociation prior to cell sorting, a process that interferes with the assessment of the disease-driven microglia transcriptomic changes (19). Finally, other studies used laser-capture approaches to analyze the transcriptomic changes in plaque-associated and/or plaque-distant tissues, thus capturing transcriptomic changes arising in heterogeneous cell types $(6,20,21)$. However, the transcriptomes of PAM and PCM have never been directly established.

To overcome these limitations, we combined cell-specific laser capture and RNA-seq analysis to investigate the functional roles of both PAM and PCM. First, we established that this approach is well suited to study the transcriptome remodeling of spatially distinct microglia. We then identified, in both microglia subpopulations, networks of co-deregulated genes and analyzed their potential functional roles in AD. Moreover, by investigating the microglia transcriptomic remodeling at early, intermediate and late stages of the disease, we were able to highlight the dynamics of these processes. Our comprehensive study confirms that proximity to Ab-plaques dramatically alters the microglia transcriptome and reveals that these changes can have both positive and negative impacts on the surrounding cellular network. Our approach also allowed us to study the overlooked plaque-distant microglia and to reveal that PCM are not bystanders to the disease progression and may be involved in Ab oligomer detection and plaque initiation, with their contribution increasing as the disease progresses.

\section{Methods}

\section{Animals}

The APPSwe/PSEN1 ${ }^{\mathrm{dE9}}$ : $\mathrm{CX} 3 \mathrm{CR} 1^{\text {+/eGFP }}$ mice, referred to as APP/PS1-CX3, used in this study were obtained

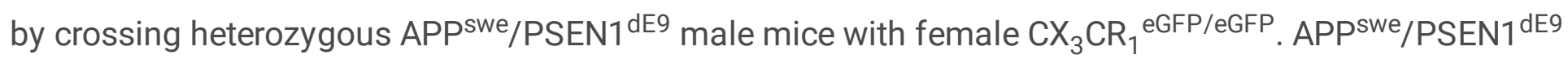
mice were purchased from the Jackson Laboratories $(22,23)$ and subsequently bred under the C57BL6/J background in the Specific Pathogen Free animal facility of the Institute for Functional Genomic (IGF, Montpellier, France, Agreement from the Ministry of Agriculture $N^{\circ}$ D34-172-13). $\mathrm{CX}_{3} \mathrm{CR}_{1}{ }^{\text {eGFP/eGFP mice }}$ were generous gift from Dan Littman (24) and were also maintained in the IGF facility under the C57BL6/J background. Comprehensive characterization of the APPswe/PSEN1 ${ }^{\mathrm{dE} 9}$ : CX3CR1 ${ }^{\text {+/eGFP }}$ has been performed previously (25) and revealed that $\mathrm{Cx} 3 \mathrm{cr} 1$ haplodeficiency has little impact on the disease progression and that APP/PS1-CX3 mice are a useful model to study microglia in AD-like pathology. Mice were housed in a $12 \mathrm{~h}$ light-dark schedule with food and water available ad libitum. All experiments followed European Union (Council directive 2010/63/UE) and institutional guidelines for the care and use of laboratory animals. The animal experiment protocols used in this study were approved by the Comité d'Ethique pour l'Expérimentation Animale Languedoc Roussillon (CEEA-LR, APAFiS\#5252). Experiments were performed in 4, 8- and 12-months-old (mo) animals (see Table S1 for details on the mice used).

\section{Tissue sample preparation}

All solutions mentioned hereon were prepared using RNAse-free buffers 
Laser microdissection. After induction of deep anesthesia with $2 \mu \mathrm{g} / \mathrm{g}$ pentobarbital (Euthasol Vet, TVM), mice were perfused intracardially with $20 \mathrm{ml}$ of phosphate buffer saline (PBS, Ambion), followed by $20 \mathrm{ml}$ PBS solution containing $20 \%$ sucrose (Sigma-Aldrich, S7903). The brain was then removed, immersed overnight in PBS solution containing $30 \%$ sucrose and then flash frozen in $-40^{\circ} \mathrm{C}$ Isopentane (Merck, \#320404). Brains were stored at $-80^{\circ} \mathrm{C}$ for at least 24 hours and for up to 6 months. $8 \mu \mathrm{m}$ thick coronal sections were cut using a cryostat (Leica) with chuck and cabin temperatures were maintained at $-24^{\circ} \mathrm{C}$. Sections were mounted onto Superfrost slides and then stored at $-80^{\circ} \mathrm{C}$ for up to two days before laser microdissection.

RNAscope: After induction of deep anesthesia with $2 \mu \mathrm{g} / \mathrm{g}$ pentobarbital (Euthasol Vet, TVM) mice were transcardiacally perfused with $10 \mathrm{ml}$ cold PBS. Brains were extracted, fixed in $4 \%$ paraformaldehyde (PFA, Sigma, P6148) for $2 \mathrm{~h}$ at room temperature (RT) and post-fixed overnight at $4^{\circ} \mathrm{C}$ in fresh $4 \%$ PFA. Tissues were then cryoprotected by successive immersion in PBS solutions containing increasing sucrose (Sigma, S7903) concentrations (i.e. $10 \%, 20 \%$ and $30 \%$ ). Tissues were transferred from one solution to the next when the brain sank indicating equilibrium between the tissue block and the solution. Brains were included in OCT (TissueTek, \#4583), flash frozen in $-50^{\circ} \mathrm{C}$ Isopentane (Merck, \#320404), and stored at $-80^{\circ} \mathrm{C}$ for at least 24 hours. $14 \mu \mathrm{m}$ thick serial coronal sections were cut using a cryostat (Leica), directly mount onto Superfrost slides and stored at $-80^{\circ} \mathrm{C}$ until use.

\section{Laser capture microdissection}

Thiazine red staining for Ab plaque detection:Thiazine red (TR) is an analog of naphthol-based azo structures which binds b-pleated sheet structures. Like Thioflavin-S, it stains dense core plaques but with maximum emission at $580 \mathrm{~nm}$ (26). On the day of microdissection, slides were removed from freezer, immediately placed for $1 \mathrm{~min}$ in $70 \%$ Ethanol solution and then stained by immersion for $1 \mathrm{~min}$ in $75 \%$ Ethanol solution containing $0.165 \%$ TR. Excess TR was removed by performing three $15 \mathrm{sec}$ with $75 \%$ Ethanol solutions. Dehydration was continued by successive immersion for $1 \mathrm{~min}$ in ethanol solutions of increasing concentration (VWR, \#20281.310, 95\%, 100\% and 100\%), followed by two immersions of 5 min each in 100\% xylene (VWR, \#289751.291). Then, slides were allowed to dry in a vacuum bell for at least one hour. Hygrometry in the microdissection room was controlled throughout the procedure and, to ensure preserving the sample quality, slides were used within $3 \mathrm{~h}$ after removal from the vacuum bell. Microglial cells (identified as GFP expressing cells) and TR staining were visualized at 20x magnification using the Nikon Eclipse Ti-E epifluorescence microscope which equipped the PixCell lle Laser Capture Microdissection system (Applied Biosystems/Excilone Elancourt, France). GFP+ cells of the cerebral cortex were laser captured in CapSure HS LCM Caps (Arcturus/Life Technologies). Laser characteristics were set at the smallest "spot" size (ie. $7.5 \mu \mathrm{m}$ ), the power of the Infra-red laser, the number and the durations of the pulses were adjusted for each slide. At the end of the session the captured cells were immediately lysed in the RLT-plus buffer (Qiagen, \#1053393) and stored at $-80^{\circ} \mathrm{C}$. In AD-CX3 mice. Plaque-associated microglia and plaque-distant microglia were isolated from the same mice with TR staining used to discriminate both microglia subtypes. Thus, PAM corresponded to microglia located within $70 \mu \mathrm{m}$ of the center of a dense core Ab plaque whereas PCM were microglia located further than 
$100 \mu \mathrm{m}$ from the center of any dense core Ab plaque (Figure S1). Distances were chosen based on the average size of the plaques (i.e. $12-20 \mu \mathrm{m}$ in diameter) and on preliminary observations showing that microglia clustered around plaques were located within 70-80 $\mu \mathrm{m}$ of the center of the plaque (not shown). Additionally, when selecting PCM we avoided microglia that, even in the absence of TR staining, appeared clustered.

\section{RNA extraction and RNA-seq}

LCM-isolated microglia from at least 4 micro-dissected sections in at least 2 independent sessions were pooled. This totaled about 400 microglial cells for each mouse and experimental condition (Table S1). RNAs were extracted using the Qiagen RNeasy Plus micro kit (Qiagen, \#74034) following a protocol slightly adapted from that of the manufacturer. RNAs were eluted in $16 \mu \mathrm{l}$ RNAse-DNAse free $\mathrm{H}_{2} \mathrm{O}$. Total RNA quality was verified by extracting RNA from the tissue remaining on the slide after microdissection and determining its integrity using the Agilent 2100 Bioanalyzer (Agilent). All RNAs had RNA Integrity Numbers (RINs) higher than 8.0.

Library preparation and RNA sequencing were performed by the ProfileXpert core facility (Lyon, France). In brief, mRNAs were pre-amplified from 200 pg total RNA using the SMART-Seq V4 Ultra Low RNA kit (Clonetec). Library preparation was performed from $500 \mathrm{pg}$ cDNAds using the Nextera kit (Illumina) following manufacturer instructions. Libraries were sequenced using an Illumina NextSeq500 platform and $75 \mathrm{bp}$ single-end sequencing data were obtained with between 28 to 41 million reads per sample. Perfect trimmed reads were aligned to Mus musculus mm10 reference genome using the TopHat2 software (27). The featureCounts tool was used to determine the number of reads mapping to each gene (28).

\section{Bioinformatics analyses and Networks}

Bioinformatics and statistical analyses were performed in collaboration with the StatABio facility (BioCampus UAR 3426 CNRS - US 09 INSERM - UM) using R software (3.6.0).

Differential gene expression: Samples to be included in the different analyses were selected according to the question addressed. After selection, gene expression normalization was performed using Relative Log Expression (RLE) normalization implemented in the $\mathrm{DESeq}_{2} \mathrm{R}$ package and genes with less than 1 count per million (cpm) in at least 3 out of 4 replicates and at least one condition, were filtered out. To detect the differentially expressed genes (DEG), we applied Generalized Linear Models (GLM) with tagwise dispersion. Both raw- $\left(\mathrm{pv}_{\mathrm{raw}}\right)$ and adjusted-Pvalues $\left(\mathrm{pv}_{\mathrm{adj}}\right)$ were computed.

Principal Component Analysis (PCA) were performed using FactoMiner R package (29).

Weighted gene co-expression network analysis (WGCNA): WGCNA R software package was applied to identify co-expression modules among pre-selected genes (30). In brief, mean connectivity and scale dependency measures were calculated to choose the proper soft power and to reconstruct the network. 
Soft threshold power was then evaluated using network analysis functions to preserve more correlated genes based on scale-free topology (31). Identification of the potential modules was performed by applying the module analysis algorithm to the dissimilarity matrix. The minimal number of genes in each module was set to limit the number of unassigned genes. In practice, unassigned genes represented 3$11 \%$ of the genes' selection. The extracted modules were labeled with colors, with turquoise being the most abundant, blue the second most abundant and brown the third most abundant modules. Unassigned genes were placed in the grey module. Eigenvalues, which can be seen as the expression values of "artificial genes" quantifying the expression variations of the genes within a particular module, were also calculated.

Gene network representations. Gene networks of preselected genes were constructed using specific applications (Apps) implemented in the Cytoscape software. First, we used the STRING App, with full STRING network and 0.7 confidence cutoff as settings to construct the network. At this stage, isolated genes were removed. Large networks were further divided into sub networks using the $M C L$ tool of the STRING App. To identify hub genes, we then ran the CytoHubba app, employing five calculation methods: Degree, Edge Percolated Component (EPC), EcCentricity, Maximal Clique Centrality (MCC), and Maximum Neighborhood Component (MNC). The intersecting genes derived using these five algorithms encode the most highly connected proteins and may represent key candidate genes with important biological regulatory functions. Finally, we used the Omics Visualizer app to display gene expression values changes on the generated networks.

Microglia gene signature enrichment: To assess the extent of which list of preselected genes overlapped with microglia gene signatures that were previously defined, we performed overrepresentation analysis (Sensome (32), DAM (11), microglial neurodegenerative phenotype (MGnD) (5), Reactome \& NewSensome, (33), IAM (34), ARM \& IRM (13), PIGs (20)). We thus determined both the enrichment factor and the associated pvalue using the Fisher-Exact-test.

Functional enrichment analysis. To study the biological mechanisms and gene ontology of the selected genes, we used the g:Profiler software (https://biit.cs.ut.ee/gprofiler/gost). The Gene Ontology (GO)biological processes associated with the selected genes were listed, nodes (GO-biological process) with adjusted $p$-value less than $5 \%$ were reported as important. To avoid overly specific and general processes, only GO-biological processes with a size between 30 and 300 were considered. When the large number of GO-terms are affected, redundancy between them is high making it difficult to read graphs. To visualize and interpret those results, we used the EnrichmentMap and AutoAnnotate apps in Cytoscape to visualize the GO-terms network (35). Contrastingly, when too few biological processes were detected, larger GObiological processes (up to 500 genes) were also considered. In these cases, to get further functional insights, Kyoto Encyclopedia Gene and Genomes (KEGG (36)) and Reactome (37) databases were also used to perform pathway enrichment analyses.

\section{RNAscope (smFISH)}


Detection of mouse Cst7, Clec7a and Cybb transcripts was performed on fixed, frozen sections using Advanced Cell Diagnostics RNAscope ${ }^{\circledR}$ Multiplex Fluorescent V2 kit. The RNAscope ${ }^{\circledR}$ Probe probes were respectively: Mm-Cst7 probe (ACD, Cat No. 498711), Mm-Clec7a (ACD, Cat No. 58264), and Mm-Cybb (ACD, Cat No. 403381). Negative controls were performed in parallel on serial sections using RNAscope ${ }^{\circledR}$ 3-plex Negative Control Probe (ACD, Cat No. 320871).

Hybridization protocol was adapted from that of ACD. In brief, slides were thawed at RT for 10 min, washed with PBS for 5 min before dehydration in 50\%, 70\% and 100\% Ethanol, 5 min each. Slides were then air-dried for 5 min and baked at $37^{\circ} \mathrm{C}$ for 1 h. $\mathrm{H}_{2} \mathrm{O}_{2}$ treatment, target retrieval, probe hybridization and signal amplification were performed according to manufacturer's instructions. Opal 570 (Perkin Elmer, $1 / 500$ in TSA buffer) was used to detect the RNAscope ${ }^{\circledR}$ probes. smFISH was followed by histological staining with Thiazine-red to detect amyloid-b plaques and immunohistochemistry with GFP to label microglia. In brief, following incubation for $1 \mathrm{~h}$ in PBS containing 20\% donkey normal serum (Gibco, S30$100 \mathrm{ml}$ ) and $0.3 \%$ Triton $X-100$ to permeabilize and block unspecific labelling, sections were incubated overnight at $4^{\circ} \mathrm{C}$ in a humidified chamber with anti-GFP (1:2000, Sigma, SAB4301138) primary antibody. Sections were then washed 3 times in PBS and incubated for $2 \mathrm{~h}$ at RT with CF488A donkey anti-rabbit (1:2000, Sigma, SAB4600036) secondary antibody. Sections were then washed 3 times 10 min with PBS and stained with $16 \mathrm{mg} / \mathrm{L}$ TR in PBS for $5 \mathrm{~min}$. After further washings with PBS to eliminate TR excess, the sections were counter stained for DAPI and mounted using ProLong Diamond antifade mountant (Invitrogen, P36961). Slides were imaged on an Imager Z1 microscope (Zeiss) equipped with an AxioCam MR R3 camera. Images were acquired with an 20X/0.50 M27 Zeiss Plan-Neofluar air objective, 11 images (corresponding to $10 \mu \mathrm{m}$-thick optical sections) were acquired.

\section{Results}

We analyzed the repertoire of genes expressed in cortical microglia from both control and AD-mice (Figure $1 \mathrm{~A}$ ) by combining laser capture microdissection (LCM) and RNA-seq approaches in transgenic mice expressing eGFP under the control of the CX3CR1 promotor $(24,25)$. Moderate tissue fixation preserves fluorescence but alters the quantity and quality of recovered RNA, whereas eGFP fluorescence is generally low in fresh unfixed brain samples (38). To overcome these issues, we developed a specific protocol based on tissue preservation by sucrose perfusion and immersion, rapid freezing in $-40^{\circ} \mathrm{C}$ isopentane, cryo-sectioning and dehydration, which allows eGFP fluorescence preservation, amyloid plaque staining as well recovery of RNA in good quality and quantity (Figure S1A).

We extracted total RNA from 400-600 microglia per animal per experimental condition (Table S1) and performed mRNA sequencing. We detected 13,923 expressed genes (Table S2), and compared the expression of 133 randomly selected ones (i.e. exhibiting low, medium or high expression levels) in FACs sorted microglia (33). Linear regression revealed a significant correlation between gene expression levels in LCM and FACS isolated microglia $(r=0.697, p<0.0001)$, demonstrating that our data are consistent with previously published microglia gene expression profiles (Figure S1B). 


\section{Isolation of microglia from mouse brain tissue with preservation of spatial information}

Cell isolation through LCM allows preservation of spatial information, but is subject to crosscontamination by surrounding cells whose processes may by be captured together with the cell of interest. To assess the degree of microglial enrichment, we evaluated the expression levels of specific microglial, astrocytic, oligodendrocytic and neuronal genes in the different LCM samples. Figure 1B shows that microglia specific genes are about 10 times enriched in the LCM samples compared to the whole cortex tissue, whereas, reversely, other glial cells and neurons specific genes are strongly depleted. In $\mathrm{CX} 3 \mathrm{CR} 1^{+/ \mathrm{eGFP}}$ mice, eGFP is expressed in all myeloid cells including infiltrating monocytes that can penetrate brain parenchyma in pathological conditions (39). To assess the possibility that our LCM samples could be contaminated by infiltrating monocytes, we analyzed the expression of peripheral monocyte/macrophage specific genes (Figure 1B). We demonstrated that the expressions of Cd163 and S100a4 were also depleted in the LCM samples. Cd74 expression was depleted in control microglia (CM), but enriched in PAM and PCM indicating that this gene is upregulated in reactive microglia.

An important drawback of cell isolation is the intrinsic cellular activation induced by generating single-cell suspension. This is particularly true for microglia which are inherently reactive cells, for which it has been shown that FACS sorting alters the analysis of the disease-induced transcriptomic changes $(19,40)$. In contrast to FACS, in LCM, cells are isolated from their environment after dehydration, which prevents cell reaction as shown by low expression of immediate early genes in the LCM isolated microglia (Figure 1C).

The reproducibility of our data was demonstrated by the strong correlation between the biological replicates (Figure S1C). In addition, principal component analysis on mRNA expression profiles allowed good discrimination between the different microglia subtypes (Figure 1D). In particular, PAM clearly separate from both CM and PCM, but remarkably we show that the PCM also separate from the CM. However, this analysis did not allow further separation of the samples by age within each sub-population. We then examined, in the different subpopulations, gene expression for markers of previously identified microglia gene signatures, namely markers of homeostatic microglia (P2ry 12 \& Tmem119, Figure 2A), Disease-associated microglia (DAM, Apoe \& Ctsd, Figure 2B, (11)), Activated-response microglia (Cst7 \& H2-ab1, ARM, Figure 2C, (13)) and Interferon-response microglia (Ifit2 \& Ifit3, IRM, Figure 2D, (13)). Our results showed that, at the difference with previous studies, homeostatic microglial gene expressions remained stable in $\mathrm{CM}$ and $\mathrm{PCM}$, and appeared to decrease only slightly in late stage PAM (Figure 2A and Table S3). Interestingly, our results revealed that DAM, ARM and IRM markers are also up-regulated in PCM with their expression increasing in an age-dependent manner (Figure 2B-D). Notably, expression of DAM and ARM markers remained stable across disease stages in PAM, whereas that of IRM appeared to increase in older PAM (Figure 2C-D).

Having established that this protocol allows the isolation of spatially distinct sub-populations of microglia with minimal intrinsic perturbation and sufficient enrichment, we conducted specific contrast analyses to identify DEGs between different sub-populations. We then performed 
bioinformatic/biostatistic analyses to infer the pathophysiological role of the different microglia subpopulations in AD progression. The workflow for data analysis is presented in Figure S2.

\section{Biological functions and Master gene regulators in PAM}

In the APP/PS1 model, dense amyloid plaques begin to appear in cortical areas at about 4-months of age (25). However, at this age, plaques are very sparse and it was not technically feasible to isolate PAM. Thus, to identify gene deregulation in PAM versus CM microglia, we restricted the analysis to 8-mo and 12-mo samples.

PCA revealed a clear distinction between CM and PAM, and statistical analysis identified 1851 DEGs (false discovery rate (FDR) $<0.05$ ), about two-thirds of which were up-regulated (Figure $3 \mathrm{~A}$ and data notshown). Previous studies established microglia reaction signatures in different pathological conditions. This includes the DAM, ARM and IRM signatures previously mentioned $(11,13)$, but also signatures for Inflammatory Associated Microglia (IAM) which represent a large set of DEGs in inflammatory conditions (34) and for the Reactome a smaller set of 86 genes deregulated in different acute and neurodegenerative conditions (33). Figure 3B shows that, compared to control microglia, PAM DEGs were significantly enriched for these different pathological microglia gene signatures. The Sensome gene signature represents a set of membrane-associated proteins and receptors that are selectively expressed in microglia and that help them sense changes in their environment (32) (refined in (33)). This signature was also significantly affected in PAM. Using a spatial transcriptomic approach, Chen et al. recently identified a plaque-induced gene (PIG) network mainly involving microglial and astroglial genes (20). As expected, PAM signature was strongly enriched with PIGs genes, actually 52 of the 57 PIGs genes were deregulated in PAM (Figure 3B).

Genes with similar expression patterns (co-expressions) are likely to have similar functions and can be grouped into modules by WGCNA (30). We performed WGCNA analysis to identify gene modules among the DEGs, and GO based enrichment analyses to extract the hypothetical biological functions for each of these modules (see Figure S2 and Materials and methods for details). Among the 1851 DEGs, we identified two distinct modules significantly correlated with the Microglia-subtype trait. The largest module (i.e. turquoise module) included 1639 genes mainly up-regulated in PAM (Figure S3A and Table $\mathrm{S} 4 \mathrm{~A})$. These genes were primarily associated with inflammation related biological processes, including Cell activation \& proliferation, Immune response, Cytotoxicity, Exocytosis, Chemotaxism, Antigen presentation, etc. (Figure 3C, Table S4B). Alterations in Cell morphology was another significantly affected biological function. The second module (i.e. blue module) was smaller and contained 212 genes mainly down-regulated in PAM (Figure S3B, Table S4A). This module mainly related to Synaptic transmission associated biological processes (Figure 3D, Table S4C).

In gene networks or subnetworks, hub genes (i.e. most highly connected genes) represent master regulators that are likely to play essential roles in controlling the biological response. We used specific applications in Cytoscape (see materials and methods) to first construct the genes' network of the two WGCNA modules, and second to identify the most connected sub-networks and their potential hub genes. 
In the Turquoise module, the 1074 most highly connected genes were separated into subnetworks using the MCC cluster tool. The ten larger clusters are detailed in Table S5, with hub genes highlighted in dark green. The three larger subnetworks are also shown in Figure S3B-D, with hub genes in yellow. In the largest subnetwork, App and Penk which are respectively up- and down-regulated in PAM appeared to play orchestrating and redundant roles for controlling chemotaxis and endopeptidase activities (Figure S3B). The second subnetwork included genes that control cell shape and antigen processing. Hub genes of this network were the GTPases Rac1, Rhoa and Rhog which belong to, respectively, the Ras and Rho super-families (Figure S3C). Rab5c is another small GTPase involved in controlling receptors endocytosis, vesicle trafficking, and endo-lysosomal pathways (41) and which played a central role in the third largest subnetwork (Figure S3D). In the blue module, we identified a single network of 48 highly connected genes (Figure S3F). This gene network was associated with control of the synaptic vesicle cycle (Table S5), hub genes were Syt1, Vamp2 and Snap25 which represent key proteins for neurotransmitter release.

Next, we addressed the question of the extent to which age affected the transcriptomic changes observed in PAM. To meet this goal, RNA-seq data from the 8-mo and 12-mo samples were reanalyzed using a Generalized Linear Model (GLM) model, with Microglia-subtype (PAM vs CM) and Age (8-mo vs 12-mo) as between samples' factors (Figure S2). Thus, we identified 723 DEG in the Microglia-subtype:Age interaction (raw p-value<0.05). Among them, we restricted our analysis to the 179 genes that were significantly deregulated in PAM vs CM (Figure S4A). WGCNA analysis further identified three genes modules (turquoise, blue and brown) that were significantly correlated with the Microglia-subtype trait. The largest module (i.e. turquoise module) included 96 genes that related to (1) Wound healing, Cell projection organization and PKB signaling biological processes, (2) Bacterial invasion and Phagosome KEGG pathways and (3) Ephrin signaling KEGG pathways (Figure S4C). Interestingly, these genes were more strongly over-expressed in 8-mo versus 12-mo PAM and were slightly over-expressed in 12-mo CM suggesting that the normally occurring overexpression of these genes during normal ageing was accelerated in PAM (Figure S4B). The blue module regrouped genes that were mostly down-regulated in PAM and tended to be less expressed / more down-regulated in 8-mo PAM. Moreover, these genes were also down-regulated in 12-mo CM compared to 8-mo CM. Genes of this module did not relate to any specific $\mathrm{GO}$ biological processes, but were associated with MAP kinases, ErbB signaling and mitophagy KEGG pathways (Figure S4C). Finally, genes of the brown module are upregulated in PAM but downregulated in older CM (Figure S4B). These genes are associated to hypoxia related and cell adhesion pathways (Figure S4C).

Overall, our comparison of PAM versus CM in the APP/PS1 model shows that PAM exhibit profound transcriptomic changes which drive an increased inflammatory reaction, support morphological changes and contribute to the degradation of synaptic support functions. Although location at the proximity of Abplaques is the most important driver for transcriptomic changes, Age contributes, but to a lesser extent, to the observed alterations.

\section{Biological functions and master gene regulators in PCM}


Because amyloid plaques relate to one of the most prominent features of the disease, studies on the role of microglia in Alzheimer's disease have often focused on PAM. Quite the reverse, PCM whose morphology is very similar to that of CM are generally overlooked (Figure S5A). However, these cells are also part of the pathological environment and we reasoned that they are likely to also contribute to the disease progression.

To investigate whether specific biological functions were altered in PCM versus control $\mathrm{CM}$, we identified genes significantly deregulated between the two conditions irrespective of age. PCA discriminated PCM from $\mathrm{CM}$ according to the second dimension (Figure S5B), and statistical analysis identified 102 DEGs (FDR $<0.05)$, the great majority (87/102) of which were up-regulated (Figure 4A, Table S6A). Interestingly, as for PAM, deregulated PCM DEGs were very significantly enriched for the different pathological microglia gene signatures (i.e. IAM, DAM, ARM, MGnD, IRM signatures), the Reactome and the Sensome signatures (Figure 4B). More surprisingly, PCM's DEGs were also highly enriched for PIG gene network. However, this may be explained by the fact that in Chen et al. (20) study, amyloid load was quantified based on 6E10 immunostaining which labels more diffuse Ab plaques. GO analyses also revealed that these genes are associated with immune related functions, including Tumor Necrosis Factor (TNF) and Cytokine production, Immune response and Antigen presentation (Figure 4C, Table S6B). Cellular reaction in this microglia subtype was also demonstrated by deregulation of functions linked to Cell differentiation and Myeloid activation. Among those DEGs, WGCNA analysis identified 2 distinct modules of co-deregulated genes (Table S6A). The largest one contained the vast majority of the DEGs (91/102) and corresponded to genes that showed an age-dependent upregulation in PCM (Figure 4D). The second module was limited to only 7 genes, including App, which by construction is over-expressed in the APP/PS1, and could not be related to a specific biological function (data not shown). On the other hand, gene network analysis identified a cluster of 49 highly connected genes that are strongly associated with the Lysosome $\left(\mathrm{p}=4.1 \times 10^{-11}\right)$, the Antigen processing \& presentation $\left(\mathrm{p}=2.4 \times 10^{-10}\right)$, and the Phagosome $\left(\mathrm{p}=7.4 \times 10^{-6}\right)$ KEGG pathways (Figure 4E). Cd68, Ctsd, H2-aa and C3ar1 represented hub genes within this network.

As shown in Figure 4A, gene expression changes were quite variable in PCM with a general trend for higher deregulation in microglia isolated from older mice. Additionally, although the expression changes were more similar within 4-mo and 12-mo samples, the inter-individual variation appeared greater in microglia isolated at in 8-mo mice. To address whether age affected the transcriptomic changes observed in PCM, we first identified 1334 genes deregulated in PCM versus CM (raw p-value<0.05), and then searched among them which ones are also deregulated in the Microglia-subtype:Age interaction (raw pvalue<0.05) (Figure S5C). We thus identified 595 genes whose expression changed in PCM in an agedepend manner. WGCNA analysis further identified two gene modules. The largest one (turquoise module) was significantly correlated with the Microglia-subtype factor. Genes of this module were upregulated in the intermediate and late stage of the disease (Figure S5D, upper panel) and related to inflammatory processes, notably Cytokine production, Antigen presentation, Myeloid cell activation and the Phagosome KEGG pathway (Figure S5E). Interestingly, these genes showed opposite regulation in CM 
being less expressed in 12-mo compared to younger cells. The second module (blue module) was significantly correlated with the Age factor and contained genes whose expression were down-regulated in an age-dependent manner specifically in PCM (Figure S5D, lower panel). Genes of the blue module related to Lipid oxidation, Organelle transport and Synaptic transmission biological processes (Figure S5E).

On the whole, these results demonstrated that although PCM are not associated to Ab plaques, and display homeostatic-like morphology, they exhibit age-dependent transcriptome alterations. These alterations are associated with important microglial functions that are typical of microglial reaction.

\section{To what extent do PAM and PCM differ?}

To further investigate the extent to which PAM and PCM differ at the transcriptomic level, we searched for genes significantly deregulated between the two microglia subtypes. Considering both the 8-mo and 12mo samples, we identified 551 DEGs (FDR < 0.05), of which 80\% $(446 / 551)$ were up-regulated in both PAM vs PCM (Figure 5A). WGCNA analysis identified a single module of 497 genes, which was significantly correlated with the Microglia-subtype trait $\left(r=0.95, p<2.10^{-8}\right)$ and more highly expressed in PAM (Figure 5B, Table S7A). These 497 genes were associated with inflammation related biological processes (Figure 5C, Table S7B) and, at least in part, overlapped with those deregulated in PCM vs CM, indicating that PCM present an intermediate reactive state between CM and PAM. However, some biological functions were specific to PAM, including Chemotaxism, Cell proliferation, Cell architecture and ROS production. By comparing the three lists of DEGs (i.e. PAM vs CM, PCM vs CM and PAM vs PCM), we also identified 11 genes that were deregulated in PCM only (Figure 5D). Globally, these genes showed significantly greater expression in PCM compared to $\mathrm{CM}$, whereas in PAM their expression was either not different or lower (i.e. 1 gene, Efnb3) than in controls (Table S8). This latter result suggests that PCM are also engaged in specific functions compared to PAM. However, these small panel of genes could not be associated to any specific biological processes (not shown).

Among the genes deregulated in PAM versus PCM, we then identified 96 genes that were changed in Microglia-subtype.Age interaction (raw p-value<0.05) (Figure S6A). WGCNA analysis refined this list to 91 co-expressed genes that were more highly expressed in PAM compared to PCM. These genes were enriched for biological processes associated with Actin filament organization, Cell migration \& differentiation, Peptidase activity (Figure S6B and Table S9) and for the KEGG Chemokine signaling pathway (Table S9). They showed opposite age-dependent regulation in PCM and PAM, and thus globally appeared less up-regulated in 12-mo PAM (Figure S6C and Figure S6D).

As a whole, these results indicate that although PAM and PCM share common signaling pathways, they are also engaged in specific biological functions. Our data also reveal different age-dependent regulations in PCM and PAM.

To further explore the relative contribution of PCM and PAM to AD, we tested whether AD risk genes were enriched among the PAM and PCM DEGs. To that purpose, we used a list of genes from a recent and 
extensive GWAS study, converting the human ID genes for their murine orthologs (42). Recent studies on polygenic risk scores have shown that genes with even small significance in GWAS carry information with regard to the risk of $A D(43)$, thus we tested for enrichment in GWAS genes at different cutoffs (Figure 5E). At all cutoffs ( $p$-values ranging from $10^{-6}$ to $10^{-2}$ ), PAM DEGs were significantly enriched for GWAS-associated AD genes thus suggesting that PAM play a key role in AD pathogenesis. In contrast, PCM DEGs were significantly enriched for GWAS AD genes only at the lowest cutoffs. This suggests that PCM contribute to AD pathogenesis, although, to a lower extent than PAM.

\section{Validation in brain tissue}

Laser microdissection can be used to isolate discrete cells from complex environments while preserving spatial information, however, the cell populations obtained by this method are not pure. To validate the cellular and spatial localization of the DEGs, we selected 3 genes (i.e. Cst7, Cybb and Clec7a) that show significant deregulation in both PAM and PCM and performed single-molecule fluorescence in situ hybridization (smFISH) with specific RNAScope probes against these targets (Figure 6 \& S7-S8). smFISH was coupled with immunofluorescent detection of microglia using anti-GFP antibody and amyloid plaques using ThiazinRed staining (Figure 6 and Figure S7). In agreement with the robust up-regulation of their mRNAs in PAM, Cst7, Clec7a and Cybb signals strongly colocalized in microglia associated with amyloid plaques (Figures 6A,C [Cst7] , S7A,C [Cybb] and S8A,C [Clec7a]). However, weaker but positive RNAscope signals for $C s t 7, C l e c 7 a$ and $C y b b$ were also observed in microglia located further than $70 \mu \mathrm{m}$ away from plaques (Figures 6A,D [Cst $],$ S7A,D [Cybb] and S8A,D [Clec7a]). We also observed a pronounced heterogeneity in the level of expression of these mRNAs in microglia, whether or not they were associated to Ab plaques. Indeed, some microglia expressed high levels of target mRNA, while neighboring microglia expressed little or no mRNA at all (Figure 6C, S7C and S8C, see arrow-heads).

\section{Discussion}

Microglia reaction in AD was first evidenced using bulk RNA-seq studies performed on purified microglia $(6,7)$. The molecular heterogeneity of microglia in this pathological context was then studied using top-down approaches (typically scRNA-seq). With these approaches, microglia subtypes are first identified based on transcriptomic similarities, their potential functions are then inferred based on geneontology analyses, and finally their location in the tissue are assessed retrospectively based on the expression of a handful of markers $(11-13,44)$. Other studies isolated PAM and/or PCM using FACS, relying on specific markers that were shown to be, on average, up-regulated in PAM. These studies then performed thorough characterization of the positive and negative populations $(16,18)$.

Herein, to decrypt alterations in microglial cells that are or not associated to Ab-plaques, we used an alternate strategy based on an unbiased bottom-up approach. We combined laser microdissection and RNA-seq to study transcriptome remodeling in these two spatially defined microglia subpopulations. Further we investigated the evolution of these alterations during the progression of the disease, from the early stage, when the plaques barely form, to the late stage, when Ab-plaque load stabilizes. We 
confirmed that dense amyloid plaques drive striking transcriptomic alterations, leading to reactive microglia that display strong inflammatory phenotypes and are less supportive to neuronal functions. Besides, our study also provides the first in depth characterization of spatially identified plaque-distant microglia (PCM), highlighting that although this microglia subtype does not show major morphological alterations, it exhibits, from the early stages, an increased inflammatory phenotype that progresses with the development of the pathology. Thus, our study reveals that PAM and PCM are both involved in AD progression, and engaged in functions which are only partially overlapping.

\section{Combination of LCM and RNA-seq provide a unique way to decipher the respective roles of discrete microglia subpopulations}

We have previously shown that $C \times 3 c r 1$ haplodeficiency does not alter disease progression in the APP/PS1 model (25). Here we demonstrate that quick TR staining and laser microdissection procedures preserves RNA quality. By isolating no less that 1'600-2'000 microglia cells from 4 mice per experimental conditions (32 mice in total), we were able to accurately analyze the transcriptome of spatially distinct microglial populations.

Although LCM does not allow to reach the level of purity of FACS, our data reveal that the large majority of cells analyzed are microglia. First, expression of different brain cell specific markers revealed a 10 -fold enrichment of microglial markers with parallel depletion in astrocyte, neuronal and oligodendrocyte markers. Second, in-situ hybridization of specific genes of interest confirmed their microglial localization. In CX3CR1//gfp mice, all myeloid cells express GFP, thus we cannot exclude that some of the microdissected cells are in fact infiltrated monocytes or peri-vascular macrophages. However, contamination by these specific cell types is likely to be low since they tend to express GFP at lower level (45), whereas we preferentially selected high GFP expressing cells for microdissection. Consistent with a strong enrichment of microglia in the LCM-isolated cells, we showed a high correlation between gene expression in LCM and FACS isolated GFP+ cells from CX3CR $1^{+/ g f p}$ mice. We also showed reduced expression of macrophage and monocyte markers in the LCM isolated cells. This finding is consistent with (1) the transcriptional remodeling observed in tissue surrounding Ab-plaques (21) and (2) recent studies in both mouse and human tissues showing that microglia are the only myeloid cells present at the vicinity of $A \beta$ deposits $(46,47)$, and endorses that the cells we analyzed were indeed microglia.

So far, most of our understanding of microglial gene expression changes in AD has come from RNAseq $(6,7)$ and scRNA-seq studies $(11-13,18,44)$. Yet, a major issue in transcriptional profiling of dissociated cells, including scRNA-seq, is the evoked transcriptional perturbations that may occur during tissue dissociation, and which may bias the true detection of disease induced transcriptional changes $(19,40)$. In LCM approach, the tissue is preserved throughout the procedure and cells are isolated from dehydrated slices which prevents any procedure driven transcriptional perturbations. Accordingly, here, we showed very low expression of immediate early genes in the isolated microglia. Although our technical approaches slightly differ, our results agree with those of Merienne et al. (48) who showed that combining LCM with RNA-seq analyses in reporter mice represents a useful

Page $15 / 29$ 
approach to decrypt cell-type specific gene expression patterns. Our results further expand the value of the approach as here we show that short staining can be added to the procedure to allow isolation and transcriptome analysis of spatially distinct cell subtypes.

$A D$ reactive microglia and $D A M / A R M$ have been associated with reduced expression of microglial homeostatic genes and loss of homeostatic functions $(5,6,11,13)$. In contrast, our results show that, across age/disease progression, gene expression of homeostatic microglial genes stays relatively stable in CM, PCM but also in PAM. Indeed, only slight down-regulation was observed in older PAM. Although these results contrast with previous analyses performed on sorted microglia $(5,6,11,13)$, they are consistent with qPCR analysis of whole cortical tissue, which shows slight down-regulation of the homeostatic genes Pr2y12 and Tgfb only in 12-mo samples (reanalysis of data in (25) and data not shown). Our results also agree with a recent study that revealed that downregulation of homeostatic microglia genes only occurs in mouse models with advanced neurodegeneration (49). Altogether, these results support that impairment of homeostatic functions occurs specifically in advanced stages PAM. They also support that brain dissociation procedures used in most studies may alter transcriptomic data and that homeostatic microglia functions are to a large extent preserved long after the histopathological disease hallmarks have appeared.

\section{Ab plaques drive prominent alterations in PAM transcriptome}

Being located close to one of the earliest and most prominent feature of AD pathology, PAM have been the subject many studies. Yet, they have mainly been investigated using either low throughput techniques (i.e. mainly imaging based, for review (50)) or indirect (i.e. top-down) medium / large throughput approaches. By combining direct isolation of PAM through LCM and RNA-seq analysis of their transcriptome, our approach provides a comprehensive understanding of molecular remodeling in this microglia subpopulation identified according to spatial criteria, without molecular or functional a priori. First, we showed that microglial proximity to amyloid plaques is the most important factor in microglia transcriptomic remodeling in this AD mouse model. These results are consistent with previous studies showing (1) enrichment, within LCM isolated plaques tissues samples, of DEG identified in aging TgCRND8 AD mice cortical brain homogenates (21), and (2) strong transcriptomic alterations in Abphagocytosing microglia (18). It is also in line with a recent spatial-transcriptomic study that revealed gradual co-expression of PIG genes as function of Ab accumulation (20).

More specifically, extending previous findings $(6,16,51)$, we showed that PAM DEGs are enriched for functions / GO-terms linked to inflammation and immune related pathways. In line with these findings, we showed that PAM DEGs are enriched for the microglial reactome signature (33). In addition to their roles in neuroinflammation processes, PAM have been associated with more neuroprotective functions in relation with Amyloid-b processing. Indeed, they have been proposed to play key roles in Ab encapsulation and in plaque compaction (50-53). Consistent with PAM clustering around plaques to form a physical barrier, we identified DEGs associated with (i) chemotaxis and cell migration and (ii) cell morphology (for example actin polymerization, actin organization, lamellipodium assembly). These later 
changes are also consistent with morphological changes commonly observed in PAM. We also identified enrichment for GO-terms linked to APP processing, phagocytosis, lysosome organization, which are consistent with an involvement of PAM in Ab clearance $(5,18)$. In addition to the above functions that were mainly associated with up-regulated genes, we also identified a module of co-repressed genes which relates, at the cellular level, to synapse establishment and functioning, and at the behavioral level with learning and memory. These microglia expressed genes may represent interesting targets to restore neuronal functioning. Together with our finding that the microglial Sensome signature (32) is also strongly altered in PAM, these results suggest that PAM lack to provide the appropriate support for correct synapse and neuronal functioning. These data are consistent with results showing lower response of PAM to damage signals (54).

PAM have been associated and often confounded with subpopulations of microglia identified by scRNAseq (DAM, ARM $(11,13)$ ) or by differential clustering (MGND (5)). Our spatial analysis of PAM, firmly establishes that PAM are strongly enriched for genes of the ARM, DAM and MGnD signatures. Consistent with their close proximity to Ab plaques, we also found a strong enrichment of PAM DEGs for the PIG signature (20). Our data also showed a strong enrichment for the IRM signature among the PAM DEGs suggesting that IRM, for which spatial location was not previously assessed (13), are also present at proximity of the Ab plaques. This remarkable phenotypic heterogeneity of microglia in spatially restricted plaque area was further evidenced by smFISH and immunostaining data showing that genes enriched in PAM are expressed at varying levels in different PAM of the same plaque. The origins of this local diversity remain to be established and may include paracrine regulation, cell memory mechanisms potentially involving epigenetic marks, and/or contact duration with the plaques. Although careful examination of the published data revealed that, for example, not all PAM are $\mathrm{MHCll}+(16)$ or that not all PAM exhibit decreased P2Y12 expression (5), to our knowledge, such local diversity around Ab-plaques has not yet been described.

Our study also provides information relative to the temporal changes in the microglial transcriptomic remodeling in PAM, focusing on early and intermediate stage of the disease. We identified three subsets of genes that are deregulated in PAM in an age dependent manner. In particular, we identified a small subset of genes that are up-regulated in PAM independently of age but decreased in aged CM. These genes are thus independent of the microglia aging processes and represent a unique signature of $A b$ plaques associated microglia. They are associated with the Syndecans Reactome pathway. Syndecans are known to bind to extracellular matrix molecules (55). Maintenance of high level of these genes throughout the disease progression may represent a protective mechanism aimed at maintaining $\mathrm{Ab}$ plaque compaction to prevent diffusion of smaller and more toxic oligomeric forms of $\mathrm{Ab}$. These genes are also associated with the KEGG Hif signaling pathway. HIF is associated with reactive oxygen species (ROS) formation, which suggests that aged PAM retain their ability to regulate ROS production. Interestingly, Hif1a signature has been recently associated with the Ab-phagocytosing microglia subtype (18). 
We also identified two other subsets, which contain genes that are respectively globally up-regulated and down-regulated in PAM and which showed same direction of changes in older control microglia. Such matching deregulations in older CM and PAM are consistent with aging and AD sharing similar molecular mechanisms, with AD representing an accelerated aging. Additionally, our finding that these subsets of genes exhibit less deregulation in older compared to younger PAM suggest that constant stimulation of microglia by external stimuli such as Ab plaques may, on the long term, lead to cell exhaustion / "burn out". This observation is consistent with results from PET studies which showed longitudinal decline of microglial reaction in some subsets of patients $(56,57)$.

\section{PCM, morphologically intact but nevertheless reactive}

In contrast to PAM, PCM have been overlooked in AD studies, and, when studied, this subpopulation was generally compared to PAM rather than CM. Here, the use of LCM provided us with the unique opportunity to comprehensively characterize PCM in $A D$, and to compare their transcriptome to both $C M$ and PAM.

We defined PCM as high GFP expressing cells located more than $100 \mu \mathrm{m}$ away from any TR+ plaque. These criteria are similar to those used by Rothman et al. (21) to identify brain tissues associated with plaques. Because thiazine-red stains dense core Ab plaques, we cannot exclude the possibility that some of the microdissected PCM were associated to more diffuse Ab deposits that are TR-but would have been $6 \mathrm{E} 10+$. However, during microdissection we paid attention to target isolated microglia and did not consider microglia showing any sort of clustering. Thus, PCM likely relate to microglia that are in contact with soluble or oligomeric Ab species or even no Ab species at all. One important observation is that although PCM overall morphology is not changed compared to control microglia (present data and (14)), this microglia subtype displayed a time-dependent increase in gene deregulation that correlates with disease progression. This dynamic deregulation mirrors the global increase of the neuroinflammatory status observed in the cortex of both mice and humans as the disease progress $(25,57)$, and parallels the increase in soluble $A b$ fractions in this $A D$ mouse line (data not shown). By showing specific deregulations in PCM, we demonstrated that this microglia subtype is not a by-stander but rather plays significant roles in AD progression.

Our results strongly suggest that, in addition to densely aggregated Ab species, microglia also react to Ab small oligomers or eventually monomers. Microglial detection and reaction to small oligomers are highly relevant to $A D$ progression as these oligomers are more bioactive at synapses and drive stronger microglia reaction (58). Our results are also consistent with data correlating tissue transcriptomic changes to $6 \mathrm{E} 10$ staining and showing a gradual increase in the network connectivity of PIG (20). Enrichment of PIG in the PCM signature supports that the expression of the so called "plaque induced genes" are in fact not restricted to dense Ab plaques. Similarly, our results show that genes deregulated in PCM are also enriched for the DAM / ARM / MGnD and IRM microglia signatures. This was further confirmed by our in-situ results showing expression of Cst7 and Clec7a, two ARMs markers, and $C y b b$ in PCM. Thus, our data revealed that although DAM / ARM / MGnD and IRM are further represented around $A b$ plaques, these microglia subtypes are likely to be involved in disease progression on a more general 
scale. Our data provide a new and comprehensive overview of the potential role of PCM in AD progression and identified several hub/key genes. Indeed, functional and gene network analysis of genes deregulated in PCM revealed that these cells are reactive, involved in the immune response notably by producing cytokines. In particular, we show that PCM are involved in antigen presentation and processing pathways. Gene network analysis identified several hub genes in this microglia subtype, namely H2aa, Cd68 and Ctsd. H2aa is part of the MHC class II protein complex and has been involved in antigen presentation (59). In control microglia H2aa is virtually not expressed, but our data show that it is already overexpressed in 4-mo PCM demonstrating that these processes are recruited at the very early stage of the disease. $\mathrm{Cd} 68$ and $\mathrm{Ctsd}$ are involved in phagocytosis and in lysosomal functions thus suggesting that not only PCM can detect Ab but they also react and switch-on clearing mechanisms. The microglial Ax/ and Merkt TAM receptor tyrosine kinases have recently been shown to be essential mediators of $A b$ plaques recognition and engulfment and it has been proposed that TAM-driven phagocytosis promotes rather than inhibits dense-core plaque development (52). $A x /$ is also up-regulated in PCM in the early/intermediate stage of the disease. This suggest that this receptor may not only be involved in plaque compaction but may also be involved in plaque formation with both processes representing tentative neuroprotective mechanisms aimed at sequestrating small, more toxic, Ab oligomers. More broadly, this supports that PCM might play an important role in early Ab plaque formation.

Our transcriptomic data also revealed that PCM display an inflammatory profile that build-up as the pathology progresses. Importantly, we showed that PCM reactive phenotype starts from the early stages when Ab load is small and plaque density low (i.e. less than 1 plaque $/ \mathrm{mm}^{2}$ re-analysis from (25)). Consistent with these results, Sierksma et al. (60) revealed up-regulation of inflammation related genes

from 4-mo in the APPswe/PS1 ${ }^{\mathrm{L} 166 \mathrm{P}} \mathrm{AD}$ mouse hippocampus and Sobue et al. (49) showed moderate early microglial dysfunction in AD precuneus in patients. These molecular findings also correlate with in vivo Positron Emission Tomography (PET) studies suggesting biphasic neuroinflammation response in patients, with the earliest peak occurring at a prodromal stage of the disease $(57,61)$. However, it remains to be determined whether the changes in the inflammation status observed at this early stage are associated with a global up-regulation of inflammatory genes in PCM or whether it corresponds to the occurrence of neuropathogenic niches.

\section{Conclusions}

Overall, here, we showed that LCM combined with RNA-seq allow to analyze transcriptome remodeling in spatially distinct cells without preconceived notions of the molecular and/or functional changes that would affect these cells. Our unique and unbiased strategy thus usefully complements previous results obtained using more tissue destructive approaches. Our data confirm and extend previous studies on the role of PAM in AD progression, they offer a comprehensive and temporal view of the molecular changes in these cells, focusing on early and intermediate stages of the disease in non-aggressive AD mouse model (i.e. as compared to the 5XFAD model that was most often used). As a whole, our results support that PAM may have both positive and negative impacts on the surrounding tissues: they may represent a 
physical barrier to prevent small Ab oligomers spreading, but are also releasing pro-inflammatory cytokines that are deleterious for the surrounding cells and are less supportive for synaptic functions. Importantly, our study reveals for the first time that, although PCM display a homeostatic-like phenotype, those microglia subtype are reactive, engaged in specific biological processes and actively participate to the disease progression. Our data suggest that these microglia sub-population may be key for detecting small $A b$ oligomers and initiating plaque formation. Although, PCM are less enriched than PAM for AD associated genes, they may represent interesting cellular targets. Indeed, one can speculate that if their protective roles (i.e. plaque formation) can be promoted while their pro-inflammatory functions are prevented, then disease progression could be impeded. Finally, we reveal further molecular heterogeneity in both PAM and PCM. Further work is needed to understand how this very local heterogeneity builds-up, to identify its dynamics, and to determine the consequences for the disease progression. Deciphering these mechanisms will allow to target specific subpopulation of microglia with the ultimate goal of promoting beneficial microglial functions and alleviating deleterious ones.

\section{Abbreviations}

Alzheimer's disease (AD), Amyloid-b (Ab): Plaque-associated microglia (PAM), Plaque distant microglia (PCM), control microglia (CM), genome wide association studies (GWAS), Disease-associated microglia (DAM), Late response microglia (LRM), Activated response microglia (ARM), Early response microglia (ERM), Interferon-response microglia (IRM), Transiting response microglia (TRM), microglial neurodegenerative phenotype (MGnD), Inflammatory Associated Microglia (IAM), plaque-induced gene (PIG), Class II major histocompatibility complex (MHCll+), Fluorescent-activated Cell sorting (FACS), Paraformaldehyde (PFA), Thiazine red (TR), RNA Integrity Numbers (RINs), Relative Log Expression (RLE), differentially expressed genes (DEG), Generalized Linear Models (GLM), Principal Component Analysis (PCA), Weighted gene co-expression network analysis (WGCNA), Edge Percolated Component (EPC), EcCentricity, Maximal Clique Centrality (MCC), Maximum Neighborhood Component (MNC), Gene Ontology (GO), Kyoto Encyclopedia Gene and Genomes (KEGG), Laser capture microdissection (LCM), false discovery rate (FDR), Generalized Linear Model (GLM), Tumor Necrosis Factor (TNF), singlemolecule fluorescence in situ hybridization (smFISH), reactive oxygen species (ROS).

\section{Declarations}

\section{Ethics approval and consent to participate}

All experiments followed European Union (Council directive 2010/63/UE) and institutional guidelines for the care and use of laboratory animals. The animal experiment protocols used in this study were approved by the Comité d'Ethique pour l'Expérimentation Animale Languedoc Roussillon (CEEA-LR, APAFiS\#5252)

\section{Consent for publication}


Not applicable

\section{Availability of Data and Materials:}

All data generated or analyzed during this study are either included in this published article or in its supplementary information files. Datasets generated during the current study are available in the Gene Expression Omnibus (GEO) repository.

\section{Competing interests:}

The authors declare they have no financial and non-financial competing interests.

\section{Fundings:}

This work was supported by France-Alzheimer [Grant AAP 2013 _ MicroMalzh]. The PhD of AL HemonnotGirard was partly supported by the Labex ICST [ANR 11-LABX-0015]. Dr C. Meersseman was supported by the FRM [....].

\section{Authors' contributions:}

A-L H-G performed RNAscope experiments and prepared the figures, CM developed the LCM-based microglia protocol and performed laser microdissection, MP and CR performed the WGCNA analyses, NL contributed to the laser microdissection, JL helped design the stud, CR made major contribution towards implementing the LCM-based microglia protocol and performed RNAseq experiments. FR funded part of the study, was a major contributor in designing the study and in writing the manuscript. $\mathrm{HH}$ : designed and funded the study, performed the bioinformatic analyses and write the manuscript.

All authors read and approved the final manuscript

Acknowledgments: We acknowledge Drs V. Compan and E. Audinat (IGF) for useful discussions while preparing this manuscript, Drs N. Crapart and Defrainaix (Excilone ${ }^{\mathrm{Inc}}$ ) for their help in setting-up the LCM protocol, The RAM-iExplore platform for help with animal breeding and T. Green for English editing.

\section{References}

1. Calderon-Garciduenas AL, Duyckaerts C. Alzheimer disease. Handbook of clinical neurology. 2017,145:325-37.

2. Hardy J, Selkoe DJ. The amyloid hypothesis of Alzheimer's disease: progress and problems on the road to therapeutics. Science. 2002,297(5580):353-6.

3. Streit WJ, Khoshbouei H, Bechmann I. The Role of Microglia in Sporadic Alzheimer's Disease. Journal of Alzheimer's disease : JAD. 2021,79(3):961-8.

4. Hansen DV, Hanson JE, Sheng M. Microglia in Alzheimer's disease. The Journal of cell biology. 2018,217(2):459-72. 
5. Krasemann S, Madore C, Cialic R, Baufeld C, Calcagno N, El Fatimy R, et al. The TREM2-APOE Pathway Drives the Transcriptional Phenotype of Dysfunctional Microglia in Neurodegenerative Diseases. Immunity. 2017,47(3):566-81 e9.

6. Orre M, Kamphuis W, Osborn LM, Jansen AH, Kooijman L, Bossers K, et al. Isolation of glia from Alzheimer's mice reveals inflammation and dysfunction. Neurobiol Aging. 2014,35(12):2746-60.

7. Wang Y, Cella M, Mallinson K, Ulrich JD, Young KL, Robinette ML, et al. TREM2 lipid sensing sustains the microglial response in an Alzheimer's disease model. Cell. 2015,160(6):1061-71.

8. Wolf SA, Boddeke HW, Kettenmann H. Microglia in Physiology and Disease. Annu Rev Physiol. 2017,79:619-43.

9. Hammond TR, Dufort C, Dissing-Olesen L, Giera S, Young A, Wysoker A, et al. Single-Cell RNA Sequencing of Microglia throughout the Mouse Lifespan and in the Injured Brain Reveals Complex Cell-State Changes. Immunity. 2019,50(1):253-71 e6.

10. Hirbec H, Rassendren F, Audinat E. Microglia Reactivity: Heterogeneous Pathological Phenotypes. Methods in molecular biology (Clifton, NJ). 2019,2034:41-55.

11. Keren-Shaul H, Spinrad A, Weiner A, Matcovitch-Natan O, Dvir-Szternfeld R, Ulland TK, et al. A Unique Microglia Type Associated with Restricting Development of Alzheimer's Disease. Cell. 2017,169(7):1276-90 e17.

12. Mathys H, Adaikkan C, Gao F, Young JZ, Manet E, Hemberg M, et al. Temporal Tracking of Microglia Activation in Neurodegeneration at Single-Cell Resolution. Cell reports. 2017,21(2):366-80.

13. Sala Frigerio C, Wolfs L, Fattorelli N, Thrupp N, Voytyuk I, Schmidt I, et al. The Major Risk Factors for Alzheimer's Disease: Age, Sex, and Genes Modulate the Microglia Response to Abeta Plaques. Cell reports. 2019,27(4):1293-306 e6.

14. Plescher M, Seifert G, Hansen JN, Bedner P, Steinhauser C, Halle A. Plaque-dependent morphological and electrophysiological heterogeneity of microglia in an Alzheimer's disease mouse model. Glia. 2018,66(7):1464-80.

15. Wendt S, Maricos M, Vana N, Meyer N, Guneykaya D, Semtner M, et al. Changes in phagocytosis and potassium channel activity in microglia of 5xFAD mice indicate alterations in purinergic signaling in a mouse model of Alzheimer's disease. Neurobiol Aging. 2017,58:41-53.

16. Yin Z, Raj D, Saiepour N, Van Dam D, Brouwer N, Holtman IR, et al. Immune hyperreactivity of Abeta plaque-associated microglia in Alzheimer's disease. Neurobiol Aging. 2017,55:115-22.

17. Navarro V, Sanchez-Mejias E, Jimenez S, Munoz-Castro C, Sanchez-Varo R, Davila JC, et al. Microglia in Alzheimer's Disease: Activated, Dysfunctional or Degenerative. Frontiers in aging neuroscience. 2018,10:140.

18. Grubman A, Choo XY, Chew G, Ouyang JF, Sun G, Croft NP, et al. Transcriptional signature in microglia associated with Abeta plaque phagocytosis. Nature communications. 2021,12(1):3015.

19. Haimon Z, Volaski A, Orthgiess J, Boura-Halfon S, Varol D, Shemer A, et al. Re-evaluating microglia expression profiles using RiboTag and cell isolation strategies. Nature immunology. 2018,19(6):63644. 
20. Chen WT, Lu A, Craessaerts K, Pavie B, Sala Frigerio C, Corthout N, et al. Spatial Transcriptomics and In Situ Sequencing to Study Alzheimer's Disease. Cell. 2020,182(4):976-91 e19.

21. Rothman SM, Tanis KQ, Gandhi P, Malkov V, Marcus J, Pearson M, et al. Human Alzheimer's disease gene expression signatures and immune profile in APP mouse models: a discrete transcriptomic view of Abeta plaque pathology. J Neuroinflammation. 2018,15(1):256.

22. Jankowsky JL, Slunt HH, Gonzales V, Jenkins NA, Copeland NG, Borchelt DR. APP processing and amyloid deposition in mice haplo-insufficient for presenilin 1. Neurobiol Aging. 2004,25(7):885-92.

23. Jankowsky JL, Slunt HH, Ratovitski T, Jenkins NA, Copeland NG, Borchelt DR. Co-expression of multiple transgenes in mouse CNS: a comparison of strategies. Biomolecular engineering. 2001,17(6):157-65.

24. Jung S, Aliberti J, Graemmel P, Sunshine MJ, Kreutzberg GW, Sher A, et al. Analysis of fractalkine receptor CX(3)CR1 function by targeted deletion and green fluorescent protein reporter gene insertion. Mol Cell Biol. 2000,20(11):4106-14.

25. Hemonnot-Girard AL, Valverde AJ, Hua J, Delaygue C, Linck N, Maurice T, et al. Analysis of CX3CR1 haplodeficiency in male and female APP(swe)/PSEN1(dE9) mice along Alzheimer disease progression. Brain Behav Immun. 2021,91:404-17.

26. Luna-Munoz J, Peralta-Ramirez J, Chavez-Macias L, Harrington CR, Wischik CM, Mena R. Thiazin red as a neuropathological tool for the rapid diagnosis of Alzheimer's disease in tissue imprints. Acta neuropathologica. 2008,116(5):507-15.

27. Trapnell C, Roberts A, Goff L, Pertea G, Kim D, Kelley DR, et al. Differential gene and transcript expression analysis of RNA-seq experiments with TopHat and Cufflinks. Nature protocols. 2012,7(3):562-78.

28. Liao Y, Smyth GK, Shi W. featureCounts: an efficient general purpose program for assigning sequence reads to genomic features. Bioinformatics. 2014,30(7):923-30.

29. Le S, Josse J, Husson F. FactoMineR: An R package for multivariate analysis. J Stat Softw. 2008,25(1):1-18.

30. Zhang B, Horvath S. A general framework for weighted gene co-expression network analysis. Stat Appl Genet Mol Biol. 2005,4:Article17.

31. Soleimani Zakeri NS, Pashazadeh S, MotieGhader H. Gene biomarker discovery at different stages of Alzheimer using gene co-expression network approach. Scientific reports. 2020,10(1):12210.

32. Hickman SE, Kingery ND, Ohsumi TK, Borowsky ML, Wang LC, Means TK, et al. The microglial sensome revealed by direct RNA sequencing. Nat Neurosci. 2013,16(12):1896-905.

33. Hirbec H, Marmai C, Duroux-Richard I, Roubert C, Esclangon A, Croze S, et al. The microglial reaction signature revealed by RNAseq from individual mice. Glia. 2018,66(5):971-86.

34. Sousa C, Golebiewska A, Poovathingal SK, Kaoma T, Pires-Afonso Y, Martina S, et al. Single-cell transcriptomics reveals distinct inflammation-induced microglia signatures. EMBO Rep. 2018,19(11). 
35. Reimand J, Isserlin R, Voisin V, Kucera M, Tannus-Lopes C, Rostamianfar A, et al. Pathway enrichment analysis and visualization of omics data using g:Profiler, GSEA, Cytoscape and EnrichmentMap. Nature protocols. 2019,14(2):482-517.

36. Ogata H, Goto S, Sato K, Fujibuchi W, Bono H, Kanehisa M. KEGG: Kyoto Encyclopedia of Genes and Genomes. Nucleic acids research. 1999,27(1):29-34.

37. Jassal B, Matthews L, Viteri G, Gong C, Lorente P, Fabregat A, et al. The reactome pathway knowledgebase. Nucleic acids research. 2020,48(D1):D498-D503.

38. Rossner MJ, Hirrlinger J, Wichert SP, Boehm C, Newrzella D, Hiemisch H, et al. Global transcriptome analysis of genetically identified neurons in the adult cortex. J Neurosci. 2006,26(39):9956-66.

39. Minogue AM. Role of infiltrating monocytes/macrophages in acute and chronic neuroinflammation: Effects on cognition, learning and affective behaviour. Prog Neuropsychopharmacol Biol Psychiatry. 2017,79(Pt A):15-8.

40. Marsh SE, Kamath T, Walker AJ, Dissing-Olesen L, Hammond TR, Young AMH, et al. Single Cell Sequencing Reveals Glial Specific Responses to Tissue Processing \&amp, Enzymatic Dissociation in Mice and Humans. bioRxiv. 2020:2020.12.03.408542.

41. Zerial M, McBride H. Rab proteins as membrane organizers. Nat Rev Mol Cell Biol. 2001,2(2):107-17.

42. Marioni RE, Harris SE, Zhang Q, McRae AF, Hagenaars SP, Hill WD, et al. GWAS on family history of Alzheimer's disease. Transl Psychiatry. 2018,8(1):99.

43. Escott-Price V, Myers AJ, Huentelman M, Hardy J. Polygenic risk score analysis of pathologically confirmed Alzheimer disease. Ann Neurol. 2017,82(2):311-4.

44. Srinivasan K, Friedman BA, Etxeberria A, Huntley MA, van der Brug MP, Foreman O, et al. Alzheimer's Patient Microglia Exhibit Enhanced Aging and Unique Transcriptional Activation. Cell reports. 2020,31(13):107843.

45. Mizutani M, Pino PA, Saederup N, Charo IF, Ransohoff RM, Cardona AE. The fractalkine receptor but not CCR2 is present on microglia from embryonic development throughout adulthood. J Immunol. 2012,188(1):29-36.

46. Reed-Geaghan EG, Croxford AL, Becher B, Landreth GE. Plaque-associated myeloid cells derive from resident microglia in an Alzheimer's disease model. J Exp Med. 2020,217(4).

47. Shukla AK, McIntyre LL, Marsh SE, Schneider CA, Hoover EM, Walsh CM, et al. CD11a expression distinguishes infiltrating myeloid cells from plaque-associated microglia in Alzheimer's disease. Glia. 2019,67(5):844-56.

48. Merienne N, Meunier C, Schneider A, Seguin J, Nair SS, Rocher AB, et al. Cell-Type-Specific Gene Expression Profiling in Adult Mouse Brain Reveals Normal and Disease-State Signatures. Cell reports. 2019,26(9):2477-93 e9.

49. Sobue A, Komine O, Hara Y, Endo F, Mizoguchi H, Watanabe S, et al. Microglial gene signature reveals loss of homeostatic microglia associated with neurodegeneration of Alzheimer's disease. Acta neuropathologica communications. 2021,9(1):1. 
50. Condello C, Yuan P, Grutzendler J. Microglia-Mediated Neuroprotection, TREM2, and Alzheimer's Disease: Evidence From Optical Imaging. Biological psychiatry. 2018,83(4):377-87.

51. Clayton K, Delpech JC, Herron S, Iwahara N, Ericsson M, Saito T, et al. Plaque associated microglia hyper-secrete extracellular vesicles and accelerate tau propagation in a humanized APP mouse model. Molecular neurodegeneration. 2021,16(1):18.

52. Huang Y, Happonen KE, Burrola PG, O'Connor C, Hah N, Huang L, et al. Microglia use TAM receptors to detect and engulf amyloid beta plaques. Nature immunology. 2021,22(5):586-94.

53. Zhao R, Hu W, Tsai J, Li W, Gan WB. Microglia limit the expansion of beta-amyloid plaques in a mouse model of Alzheimer's disease. Molecular neurodegeneration. 2017,12(1):47.

54. Gyoneva S, Swanger SA, Zhang J, Weinshenker D, Traynelis SF. Altered motility of plaque-associated microglia in a model of Alzheimer's disease. Neuroscience. 2016,330:410-20.

55. Xian X, Gopal S, Couchman JR. Syndecans as receptors and organizers of the extracellular matrix. Cell Tissue Res. 2010,339(1):31-46.

56. Blume T, Focke C, Peters F, Deussing M, Albert NL, Lindner S, et al. Microglial response to increasing amyloid load saturates with aging: a longitudinal dual tracer in vivo muPET-study. J Neuroinflammation. 2018,15(1):307.

57. Fan Z, Brooks DJ, Okello A, Edison P. An early and late peak in microglial activation in Alzheimer's disease trajectory. Brain : a journal of neurology. 2017,140(3):792-803.

58. Yang T, Li S, Xu H, Walsh DM, Selkoe DJ. Large Soluble Oligomers of Amyloid beta-Protein from Alzheimer Brain Are Far Less Neuroactive Than the Smaller Oligomers to Which They Dissociate. J Neurosci. 2017,37(1):152-63.

59. Roche PA, Furuta K. The ins and outs of MHC class Il-mediated antigen processing and presentation. Nature reviews Immunology. 2015,15(4):203-16.

60. Sierksma A, Lu A, Mancuso R, Fattorelli N, Thrupp N, Salta E, et al. Novel Alzheimer risk genes determine the microglia response to amyloid-beta but not to TAU pathology. EMBO molecular medicine. 2020,12(3):e10606.

61. Hamelin L, Lagarde J, Dorothee G, Potier MC, Corlier F, Kuhnast B, et al. Distinct dynamic profiles of microglial activation are associated with progression of Alzheimer's disease. Brain : a journal of neurology. 2018,141(6):1855-70.

\section{Figures}

\section{Figure 1}

Study design and validation of the approach. (A) Schematic representation of the study design. (B) Gene expression of cell type markers for microglia, astrocytes, oligodendrocytes, neurons and peripheral 
immune cells in laser captured control microglia from CX3CR1 ${ }^{\text {+eGFP }}$ mice (CM, black), parenchymal microglia (PCM, grey) or plaque associated microglia (PAM, light grey) from APP/PS $1^{\mathrm{Tg} / 0}: \mathrm{CX} 3 \mathrm{CR} 1^{+/ \mathrm{eGFP}}$ mice. Gene expression is normalized to housekeeping genes expression and set at 1 in whole cortex homogenates. (C) Gene expression of immediate early genes in the microglial sub-populations. Gene expression is normalized to housekeeping genes expression and set at 1 in whole cortex homogenates. (D) Principal component analysis (PCA) of gene expression in the different microglia samples, based on the 13,923 expressed genes.

\section{Figure 2}

Gene expression of specific microglial genes in the different microglia subpopulations (A) Homeostatic genes, (B) DAM genes, (C) ARMs genes, (D) IRM genes. Control microglia (CM, greens), APP/PS1 parenchymal microglia (PCM, oranges) and plaque associated microglia (PAM, purples). Shades code for age: 4-mo, light color, 8-mo, median color, and $12 \mathrm{mo}$, dark color.

\section{Figure 3}

Microglial gene expression remodeling in plaques associated microglia (A) Heatmap of differentially expressed genes in PAM (violets) versus CM (greens) along aging. The scaled expression value (row Z score) is shown in a blue-red color scheme with red indicating higher expression, and blue lower expression. The full data is available in Table S2A. (B) Enrichment in DEGs in different gene signatures. The size and the color of each dot are respectively proportional to the number of DEGs and the adjusted p-value. The number of DEGs is also indicated in or at proximity of the dot. (C-D) Cytoscape representation of deregulated GO terms ("Biological Process" category) in the Turquoise (C) and Blue (D) gene modules. The full data is available in TableS2B-C. Each dot represents a deregulated GO-Term, their size and color are respectively proportional to the number of genes in the GO-term and the enrichment adjusted p-value. GO-Terms are grouped into categories using the Autoannotate Cytoscape app.

\section{Figure 4}


Microglial gene expression remodeling in parenchymal AD microglia. (A) Heatmap of differentially expressed genes in PCM (Oranges) versus CM (greens) along aging. The scaled expression value (row $Z$ score) is shown in a blue-red color scheme with red indicating higher expression, and blue lower expression. The full data is available in Table S4A. (B) Enrichment in DEGs in different gene signatures. The size and the color of each dot are respectively proportional to the number of DEGs and the adjusted p-value. The number of DEGs is also indicated in or at proximity of the dot. (C) Cytoscape representation of deregulated GO terms ("Biological Process" category) in PCM versus CM DEGs. The full data is available in Table S4B. Each dot represents a deregulated GO-Term, their size and color are respectively proportional to the number of genes in the GO-term and the enrichment adjusted p-value. GO-Terms are grouped into categories using the Auto-annotate Cytoscape app. (D) Boxplots of eigengene values for each sample in the most abundant WGCNA module of PCM versus CM DEGs (91 genes). Statistical analyses: 2-ways ANOVA with Microglia subtype and Age as between subjects' factors. Subtype $p<0.001$,

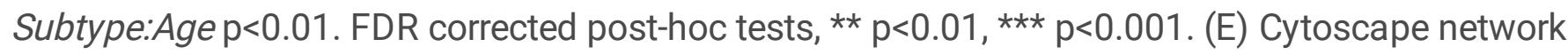
visualization of highly connected genes in PCM versus CM DEGs. Log $_{2}$ expression ratio between PCM and $\mathrm{CM}$ are mapped to the nodes using a blue-white-red gradient with red indicating higher expression in PCM, and blue lower expression. The inner, middle and outer rings represent Log-ratio in respectively the 4-, 8- and 12-mo samples. Yellow nodes depict hub genes within the network.

\section{Figure 5}

\section{Microglial gene expression remodeling between plaques associated and parenchymal AD microglia. (A)}

Heatmap of differentially expressed genes in PAM (violets) versus PCM (oranges) along aging. The scaled expression value (row $Z$ score) is shown in a blue-red color scheme with red indicating higher expression, and blue lower expression. The full data is available in Table S5A. (B) Boxplots of eigengene values for each sample in the most abundant WGCNA module of PAM versus PCM DEGs (497 genes). Statistical analyses: 2-ways ANOVA with Microglia subtype and Age as between subjects' factors. Subtype $p<0.001$, Subtype:Age $p<0.05$. FDR corrected post-hoc tests, $* \star \star ~ p<0.001$. (C) Cytoscape representation of deregulated GO terms ("Biological Process" category) in the most abundant module of PAM versus PCM DEGs (497 genes). The full data is available in Table S5B. (D) Venn diagram showing the overlap between DEGs in PAM versus $C M$ ( $F D R<0.05$, violet circle), $P C M$ versus $C M$ ( $F D R<0.05$, orange circle), and PAM versus PCM (FDR<0.05, green circle). (E) Bar plot showing the significance (- $\log 10$ (pvalue)) of enrichment of AD GWAS genes in PAM and PCM DEGs. Different AD GWAS sets corresponding to different p-value cutoffs (Marioni al., 2018), were tested. The numbers under brackets indicate the number of ortholog genes at the cutoff, and the numbers in columns, the numbers of PAM or PCM DEG GWAS genes. 


\section{Figure 6}

Cst7 mRNA expression in control (CM), parenchymal (PCM) and plaque-associated (PAM) microglia. Microglia detection (GFP, green), Thiazine Red (ThR) staining for amyloid plaques (red) and Cst7 mRNA expression (purple) were combined on brain slices in (A) APP/PS1 $1^{\mathrm{Tg} / 0}: \mathrm{CX} 3 \mathrm{CR} 1^{+/ \mathrm{eGFP}}$ or (E) $\mathrm{CX} 3 \mathrm{CR} 1^{+/ \mathrm{eGFP}}$ mouse. (A) 12-mo APP/PS1 ${ }^{\mathrm{Tg} / 0}: \mathrm{CX} 3 \mathrm{CR} 1^{\text {+/eGFP }}$ mouse cortical field exhibiting three amyloid plaques. Plaque associated microglia (PAM) are located within $70 \mu \mathrm{m}$ (purple dotted circle) of the plaque epicenter, while parenchymal microglia (PCM) are located farther than $100 \mu \mathrm{m}$ (orange dotted circle) from the plaque epicenter. Cst7 mRNA expression is absent from control microglia $(E)$ but can be evidenced in both plaque-associated (A, C) and parenchymal (A, D) microglia. White arrowheads point to Cst7 positive parenchymal microglia. (B) Pseudocounts of Cst7 expression in CM (green), PCM (orange) and PAM (violet) along ages. (C) Zoomed region around ThR+ plaque showing Cst7 overexpression in PAM. Note the heterogeneity for Cst7 expression amongst PAM, blue arrowhead for no expressing microglia, yellow arrowhead for low expressing microglia, grey arrowhead for high expressing microglia. (D) Zoomed region of Cst7 positive parenchymal microglia. Scale bar $20 \mu \mathrm{m}$.

\section{Supplementary Files}

This is a list of supplementary files associated with this preprint. Click to download.

- FigSupp1Hemonnot.eps

- FigSupp2Hemonnot.eps

- FigSupp3Hemonnot.eps

- FigSupp4Hemonnot.eps

- FigSupp5Hemonnot.eps

- FigSupp6Hemonnot.eps

- FigSupp7Hemonnot.eps

- FigSupp8Hemonnot.eps

- HemonnotGirard2021AdditionalTable1.xlsx

- HemonnotGirard2021AdditionalTable2.xIsx

- HemonnotGirard2021AdditionalTable3.xlsx

- HemonnotGirard2021 AdditionalTable4.xIsx

- HemonnotGirard2021 AdditionalTable5.xlsx

- HemonnotGirard2021AdditionalTable6.xlsx

- HemonnotGirard2021AdditionalTable7.xlsx

- HemonnotGirard2021 AdditionalTable8.xlsx 
- HemonnotGirard2021 AdditionalTable9.xIsx 